\title{
Transcription Factor MafB Promotes Hepatocellular Carcinoma Cell Proliferation through Up-Regulation of Cyclin D1
}

\author{
Hao Yu Hong-lei Jiang Dong Xu Jun-zhe Jin Zhe-ming Zhao Yan-dong Ma \\ Jian Liang \\ Department of general surgery, the fourth affiliated hospital of China medical university, Shenyang, \\ China
}

\section{Key Words}

Hepatocellular carcinoma $•$ Cell proliferation $•$ MafB $•$ Cyclin D1

\begin{abstract}
Background/Aims: MafB, a member of the Maf transcription factor family, plays a key role in the regulation of pancreatic alpha and beta cell differentiation. However, its function in the control of cancer cell proliferation remains unknown. Methods: The mRNA and protein expression levels of MafB in hepatocellular carcinoma tissues and adjacent non-tumor normal specimens were determined by real-time RT-PCR and Western blot, respectively. Report assay was performed to determine whether the regulation of Cyclin D1 by MafB is at the transcriptional level. The binding of MafB to the Cyclin D1 promoter was determined by Chromatin Immunoprecipitation (ChIP) assays. To determine the potential oncogenic effects of MafB in vivo, HepG2 cells transfected with adenovirus containing empty vector or MafB were injected subcutaneously to the skin under the front legs of the nude mice. Results: In the current study, we showed that MafB was markedly up-regulated in hepatocellular carcinoma (HCC) tissues and cells. Enforced overexpression of MafB enhanced, while its deficiency inhibited HCC cell proliferation. Mechanistically, Cyclin D1, an important regulator of cell cycle progression, was identified as a direct transcriptional target of MafB. Consistently, knockdown of Cyclin D1 largely attenuated the proliferative roles of MafB in HCC cells. Importantly, MafB overexpression significantly promoted cancer cell growth in mice. Conclusions: Collectively, our results identified a novel HCC regulatory pathway involving MafB and Cyclin D1, the dysfunction of which drives proliferative character in HCC.
\end{abstract}

(C) 2016 The Author(s)

Published by S. Karger AG, Basel

\section{Introduction}

Hepatocellular carcinoma (HCC) has become one of the most prevalent malignancies and leading causes of death in China [1]. Although some recent progress has been made with regard to understand the genetic alterations in HCC development [2], its molecular mechanisms remain to be elucidated. 


\section{Cellular Physiology Cell Physiol Biochem 2016;39:700-708

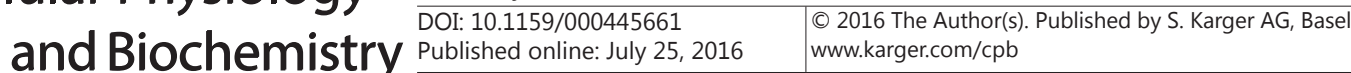 \\ Yu et al.: MafB Promotes HCC Proliferation through Cyclin D1}

V-maf musculoaponeurotic fibrosarcoma oncogene homologue B (MafB), belonging to basic leucine zipper transcription factor, is a widely expressed and involved in several biological events [3, 4]. Initially, MafB was shown to form heterodimers with v-Maf and Fos through its zipper structure and induced transformation of chicken embryo fibroblasts in vitro [5]. Subsequent studies found that MafB played an important role in the regulation of pancreatic alpha cell, beta cell, osteoclast, macrophage and adipocyte differentiation [6$10]$. Besides, MafB was viewed as a highly sensitive and specific marker for poor prognosis in multiple myeloma patients [11]. Moreover, silencing of MafB inhibited migration and invasion abilities in nasopharyngeal carcinoma cells [12], suggesting that MafB may also be of significance for cancer biology.

Herein, we analyzed expression of MafB in human HCC tissue samples and found that mRNA and protein levels of MafB were significantly elevated in HCC tissues, compared with adjacent normal tissues. Besides, ectopic MafB expression in HCC cells enhanced cell proliferation and invasion, whereas MafB knockdown suppressed the phenotypes. Therefore, we provide direct evidence that MafB could be an oncogene in tumorigenesis and it can be a potential therapeutics to treat HCC.

\section{Materials and Methods}

\section{Human tissue samples}

20 parried of HCC tissues and adjacent non-tumor normal specimens were collected from routine therapeutic surgery at our department. Informed consent was obtained from each patient before surgery. The study protocol was reviewed and approved by the Institutional Review Board of the fourth affiliated hospital of China medical university

Generation of recombinant adenoviruses

Recombinant adenovirus expressing human MafB or empty vector (EV) was generated using the AdEasy ${ }^{\mathrm{TM}}$ Adenoviral Vector System (Qbiogene, Irvine, CA, USA) according to the manufacturer's instructions.

Cell culture, transfection and luciferase assays

HCC cells were obtained from The Cell Bank of Type Culture Collection of Chinese Academy of Sciences (CAS, Shanghai). Cells were grown in Dulbecco's modified Eagle's medium (Invitrogen, Grand Island, NY, USA) supplemented with $10 \%$ fetal bovine serum (Invitrogen) and maintained at $37^{\circ} \mathrm{C}$ in a humidified atmosphere with $5 \% \mathrm{CO}_{2}$. RNAi-mediated depletion of MafB or Cyclin D1 was achieved by infecting cells with small interfering RNA (siRNA) oligos (GE Dharmacon, Pittsburgh PA, United States). Human Cyclin D1 promoter from $-1000 \mathrm{bp}$ to $+1 \mathrm{bp}$ was amplified by PCR and inserted into the KpnI and XhoI sites of PGL4.15 empty vector (Promega, Madison, WI, USA). Mutant MafB binding site was generated using a PCR mutagenesis kit (Toyobo). All the transfection experiments were performed when cells were grown to $70 \%$ to $80 \%$ confluence, using Lipofectamine 2000 reagents (Invitrogen) according to the manufacturer's instructions. For luciferase assays, cells were seeded in 24-well plates and transfection efficiency was normalized by co-transfecting Simian virus 40 (SV40) plasmids (Promega). Luciferase values were measured using the Dual-Luciferase Reporter Assay System (Promega).

\section{BrdU incorporation assays}

A cell proliferation enzyme-linked immunosorbent assay (BrdU kit; Beyotime) was used to analyze the incorporation of BrdU during DNA synthesis following the manufacturer's protocols. Absorbance was measured at $450 \mathrm{~nm}$ in the Spectra Max 190 ELISA reader (Molecular Devices, Sunnyvale, CA).

Flow cytometry analysis

Cells were trypsinized and fixed in $70 \%$ ethanol at $-20^{\circ} \mathrm{C}$ overnight. Cells were then resuspended in PBS containing $40 \mu \mathrm{g} / \mathrm{ml}$ propidium iodide and $100 \mu \mathrm{g} / \mathrm{ml}$ RNase A. After incubation for $1 \mathrm{~h}$ at $37^{\circ} \mathrm{C}$, cells were characterized and cell cycle distribution was determined by fluorescence activated cell sorting (FACS). For each sample, $2 \times 10^{4}$ cells were analyzed. Data was acquired using a BD LSRII apparatus and analyzed using the FlowJo software. 


\section{Cellular Physiology Cell Physiol Biochem 2016;39:700-708 \begin{tabular}{l|l} 
DOI: 10.1159/000445661 & $\begin{array}{l}\text { O 2016 The Author(s). Published by S. Karger AG, Basel } \\
\text { www.karger.com/cpb }\end{array}$
\end{tabular} \\ Yu et al.: MafB Promotes HCC Proliferation through Cyclin D1}

Colony formation assays

HepG2 cells were seeded in a 6-well plate 48 hours post-transfection and cultured for 8 to 10 days at $37^{\circ} \mathrm{C}$ in $5 \% \mathrm{CO}_{2}$. Cells were fixed with $4 \%$ paraformaldehyde in phosphate-buffered saline (PBS), washed twice with PBS, and stained with a crystal violet solution (1\% crystal violet, $10 \%$ ethanol in water). Stained cells were washed thrice with water and counted by under an optical microscope.

\section{Mouse Experiments}

Male BALB/c nude mice aged 6 weeks were purchased from Shanghai Laboratory Animal Company (SLAC, Shanghai). $4 \times 10^{6} \mathrm{HepG} 2$ cells stably expressing MafB or negative controlwere injected subcutaneously to the skin under the front legs of the nude mice. The mice were observed over 16 days for tumor formation. Mice were then sacrificed and the wet weights of each tumor were determined.

\section{Real-time PCR analysis}

Total RNA from tissues and cells was extracted using the TRIzol Kit (Invitrogen) according to the manufacturer's instructions. cDNA was transcribed from $1 \mu \mathrm{g}$ of total RNA following the manufacturer's instructions (Promega). Quantitative real-time PCR was performed using SYBR Premix Ex Taq reagents (Takara, Shiga, Japan). Relative transcript quantities were calculated using the $2^{-\Delta \Delta} \mathrm{Ct}$ method with $\beta$-actin as the endogenous reference gene.

\section{Western blots}

Cells and tissues were harvested and lysed with ice-cold lysis buffer (50 mM Tris- $\mathrm{HCl}, \mathrm{pH} 7.4,100$ $\mathrm{mM}$ 2-Mercaptoethanol, 2\% w/v SDS, $10 \%$ glycerol). After centrifugation at 10,000 $\mathrm{g}$ for $10 \mathrm{~min}$ at $4{ }^{\circ} \mathrm{C}$, proteins in the supernatants were quantified, separated by $10 \%$ SDS PAGE and transferred to polyvinylidene difluoride (PVDF) membranes (Millipore, Bedford, MA, USA). The membranes were blocked with 10\% nonfat milk and then incubated with different primary antibodies, followed by incubation with horseradish peroxidase-conjugated secondary antibodies. The following primary antibodies were used: anti-MafB (Santa Cruz, sc-376387, 1:1000); Cyclin D1 (Santa Cruz, sc-753, 1:2000). Protein levels were normalized to total $\beta$-actin (Santa Cruz, sc-47778, 1:2500). The proteins were visualized by an ECL chemiluminescence detection kit (Amersham Biosciences, Buckinghamshire, UK).

\section{Statistical analysis}

Data were expressed as mean \pm standard error of the mean (SE). Analysis was conducted with GraphPad Prism version 5.01 (GraphPad Software). Significance between two groups was analyzed using the unpaired 2 -tailed $t$ test $\left({ }^{*} \mathrm{p}<0.05,{ }^{* *} \mathrm{p}<0.01,{ }^{* * *} \mathrm{p}<0.001\right)$.

\section{Results}

MafB is up-regulated in HCC

To date, the expression of MafB in HCC tissues has not been investigated. Therefore, its mRNA level was determined in 20 primary HCC and adjacent normal specimens using quantitative real-time PCR. As a result, we observed a markedly up-regulation of MafB expression levels in HCC tissues (Fig. 1A), which was further confirmed by Western blots (Fig. 1B). The expression of MafB was also significantly increasedacross a panel of HCC cell lines (HepG2, Hep3B, HuH7, HCCC-9810, BEL-7402 and QGY-7703) compared to normal liver epithelial cells (THLE3) (Fig. 1C).

\section{Overexpression of MafB promotes HCC cell proliferation}

Next, to evaluate the functional outcomes of MafB in HCC progression, adenovirus containing empty vector (EV) or MafB was administrated into HepG2 cells (Fig. 2A). Then, cell growth was assessed. The results showed that cells overexpressing MafB had significantly increased growth rates and proliferative abilities (Fig. 2B-2C). Cell colony formation in soft agar was also increased by MafB overexpression (Fig. 2D). Moreover, the percentage of cells in the $S$ phase was significantly higher for MafB-expressing cells (Fig. 2E), suggesting the 
A

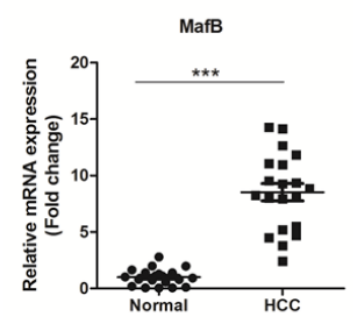

B

Patient 1

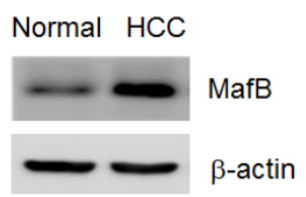

C

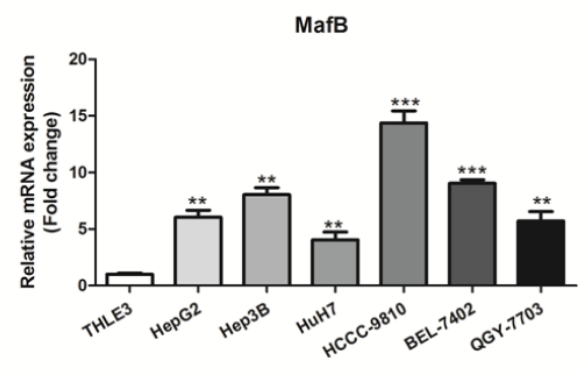

Patient 2

Normal HCC

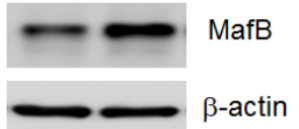

Fig. 1. Up-regulation of MafB in HCC. (A) Relative mRNA levels of MafB were determined by quantitative real-time PCR analysis in 20 primary HCC and adjacent normal liver tissues. (B) Representative protein levels of MafB in HCC and normal liver tissues from three patients. (C) Relative mRNA levels of MafB in HCC cells and normal liver epithelial cells (THLE3). ${ }^{* *} P<0.01,{ }^{* * *} P<0.001$ between two groups.

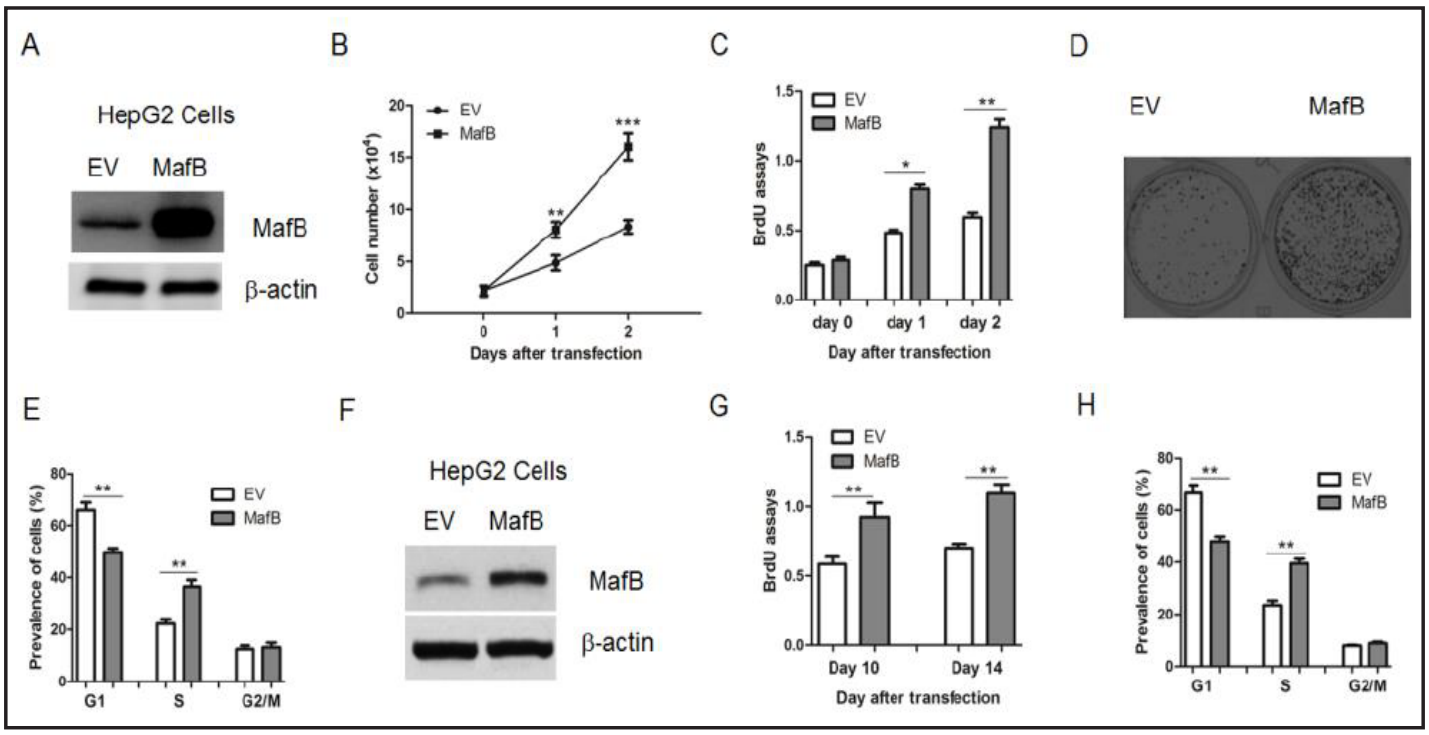

Fig. 2. MafB promotes HCC growth in vitro. (A) Representative protein levels of MafB in HepG2 cells transfected with adenovirus containing empty vector (EV) or MafB for 24 h. (B-C) Cell number (B) and cell proliferation (C) in HepG2 cells expressing empty vector (EV) or MafB. (D) Representative anchorage-independent growth activity for HepG2 cells with ectopic MafB expression and its corresponding control (EV). The colonies were observed at lower magnification ( $40 \mathrm{x}$ ). (E) Cell-cycle analysis in HepG2 cells expressing empty vector (EV) or MafB. (F) Representative protein levels of MafB in HepG2 cells transfected with lentivirus containing empty vector (EV) or MafB. 10 days after transfection, cells were subjected to western blot analysis (F-H). (G-H) Cell proliferation (G) and cell-cycle analysis (H) in HepG2 cells expressing empty vector (EV) or MafB. 10 or 14 days after transfection, cells were subjected to analysis. ${ }^{*} P<0.05,{ }^{* *} P<0.01,{ }^{* * *} P<$ 0.001 between two groups.

acceleration of the G1/S transition. Given that adenoviruses may assure high transfection efficiency without integration in the genome of the target cells, lentiviruses were used. HepG2 cells were transfected with lentiviruses containing MafB or empty vector (EV) and KARGER 
A

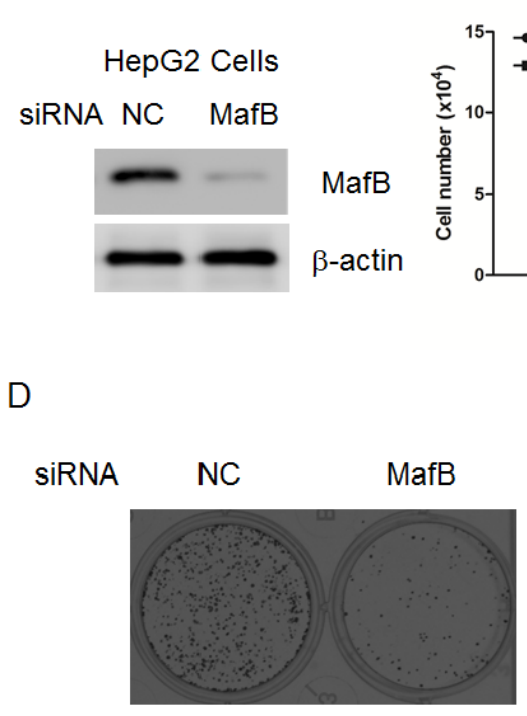

B

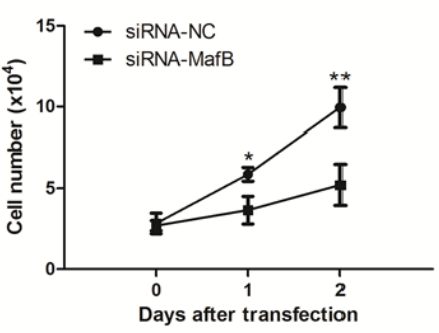

$\mathrm{E}$
C

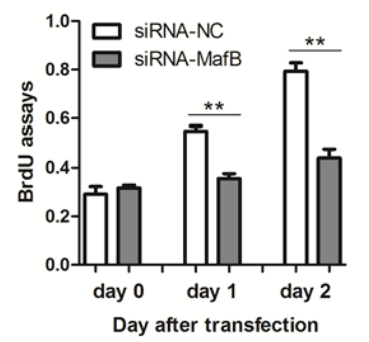

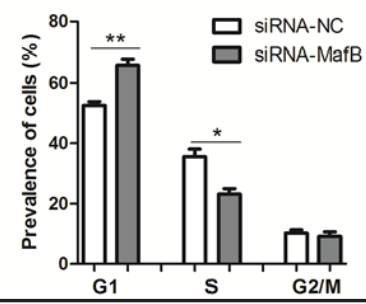

Fig. 3. Knockdown of MafB inhibits HCC cell proliferation. (A) Representative protein levels of MafB in HepG2 cells transfected with siRNA oligos targeting MafB or negative control (NC) for $24 \mathrm{~h}$. (B-C) Cell number (B) and cell proliferation (C) in HepG2 cells. (D) Representative anchorage-independent growth activity for HepG2 cells. The colonies were observed at lower magnification ( $40 \mathrm{x}$ ). (E) Cell-cycle analysis in HepG2 cells transfected with siRNA oligos targeting MafB or negative control (NC). ${ }^{*} P<0.05,{ }^{* *} P<0.01$ between two groups.

cell proliferation were analyzed 10 or 14 days after transfection (Fig. 2F). In agreement, lentivirus-mediated overexpression of MafB also led to an enhanced cell proliferation and accelerated cell-cycle (Fig. 2G-2H).

Reciprocally, knockdown of MafB by small interfering RNA (siRNA) oligos in HepG2 cells suppressed proliferation, colony formation and G1/S transition (Fig. 3A-3E). Therefore, our results indicate that MafB promotes tumor cell proliferation in vitro.

\section{Identification of Cyclin D1 as a direct target of MafB}

The finding of a critical role of MafB in the cell-cycle progression and cell proliferation prompted us to identify its potential down-stream target gene associated with the G1/S transition. As a result, mRNA level of Cyclin D1, but not other regulators of cell-cycle entry, was increased in cells overexpressing MafB (Fig. 4A), which was confirmed by western blots analysis (Fig. 4B). Consistently, knockdown of MafB reduced the expression of Cyclin D1 in HepG2 cells (Fig. 4C and 4D). In addition, we observed a significant positive correlation between MafB and Cyclin D1 expression in HCC and normal liver specimens (Fig. 4E), further suggesting that Cyclin D1 is a direct transcription target of MafB in tumorigenesis.

\section{Positive regulation of Cyclin D1 promoter by MafB}

To better gain insights into the molecular basis of up-regulation of Cyclin D1 by MafB, the promoter region of human Cyclin D1 gene was cloned into a luciferase reporter vector. As a result, the promoter activity of Cyclin D1 was increased by MafB overexpression in a dose-dependent manner (Fig. 5A). By using two software programs (TFSEARCH and TESS), a potential regulatory site containing MafB binding site was also identified, which located at -372 bp (Fig. 5B). Indeed, the Cyclin D1 promoter with the mutant site failed to be activated by MafB overexpression (Fig. 5B). The luciferase activity containing the truncated Cyclin D1 promoter construct was also not induced by MafB (Fig. 5C), suggesting that this site is essential for MafB-activated Cyclin D1 transcription. The direct interaction of MafB with the endogenous Cyclin D1 promoter was also detected by chromatin immunoprecipitation (ChIP) 
A

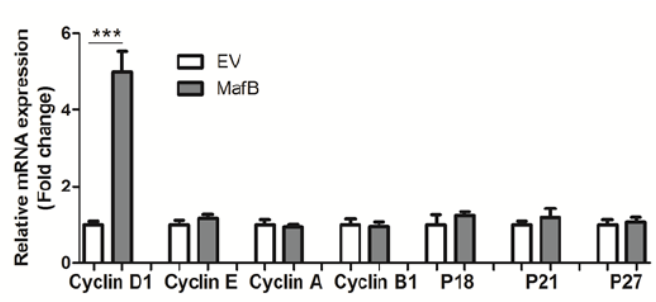

B

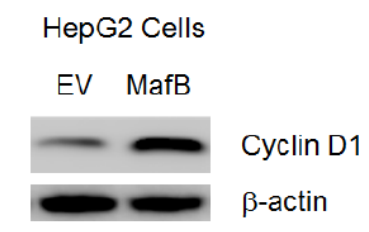

C

D

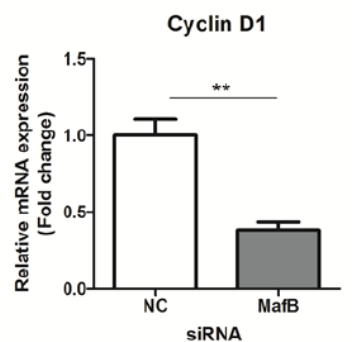

HepG2 Cells siRNA NC MafB

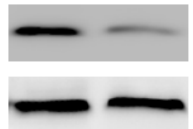

Cyclin D1

$\beta$-actin
E

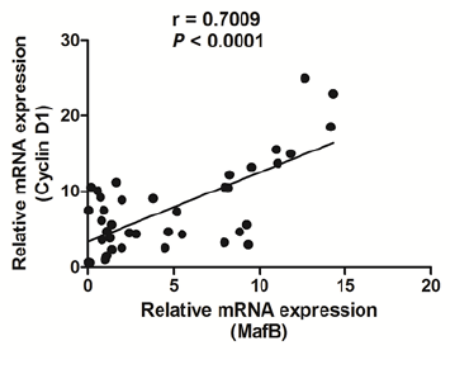

Fig. 4. MafB regulates Cyclin D1 expression in HCC cells. (A) Relative mRNA levels of Cyclin D1, Cyclin E, Cyclin A, Cyclin B1, P18, P21 and P27 in HepG2 cells overexpressing MafB or empty vector (EV). (B) Representative protein levels of Cyclin D1 in HepG2 cells. (C-D) Relative mRNA (C) and protein (D) levels of Cyclin D1 in HepG2 cells transfected with siRNA oligos targeting MafB or negative control (NC). (E) A positive correlation between MafB and Cyclin D1 expression in 20 pairs of HCC and normal liver tissues. ${ }^{* *} P<0.01$, *** $P<0.001$ between two groups.

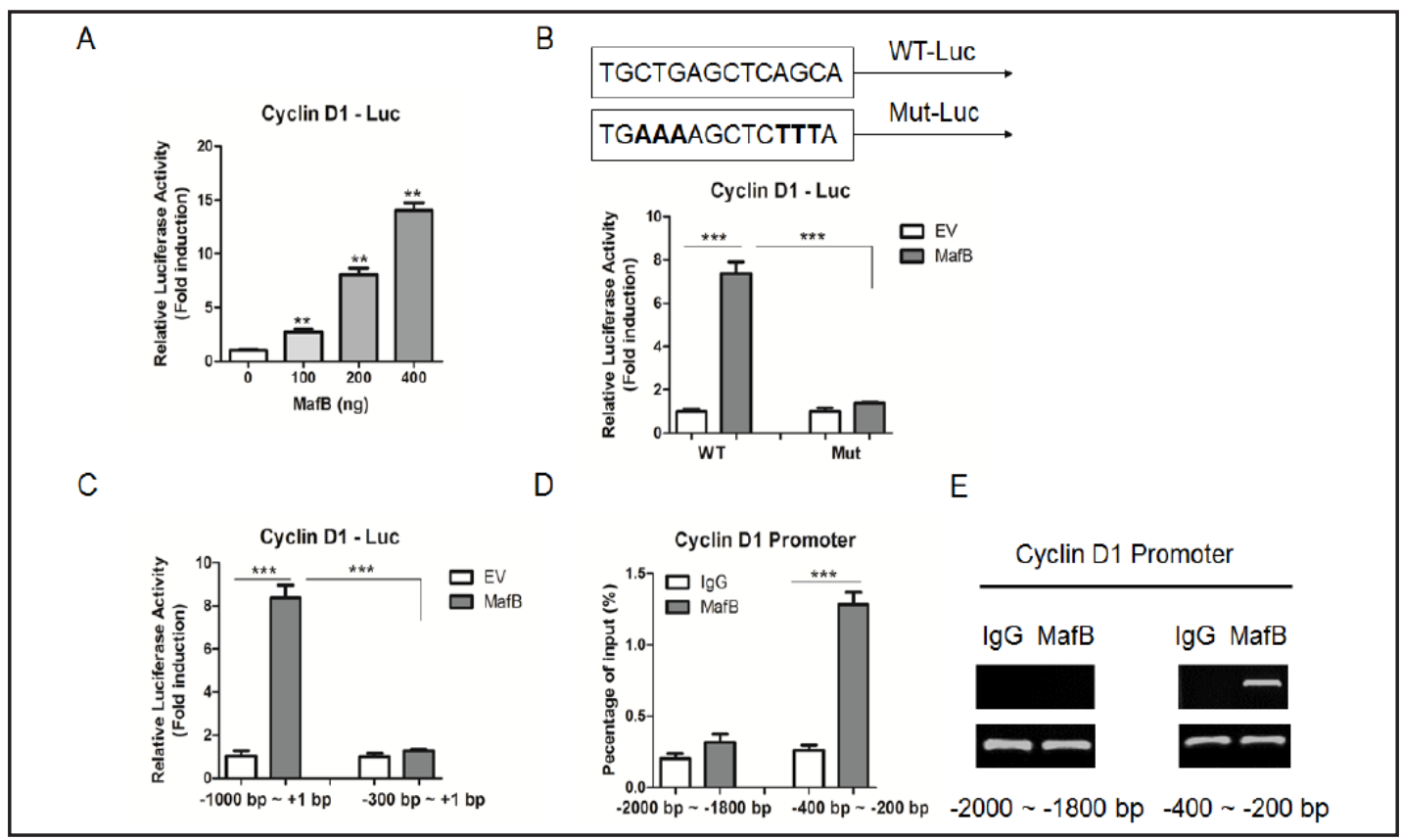

Fig. 5. Direct regulation of Cyclin D1 promoter by MafB. (A) The transcriptional activity of the Cyclin D1 promoter. HepG2 cells were co-transfected with Cyclin D1 promoter reporter and MafB expression plasmids as indicated. (B) The Cyclin D1 promoter constructs containing a potential MafB motif. Point mutations were highlighted in bold. (C) The luciferase reporter activity of two Cyclin D1 promoter constructs in HepG2 cells. (D-E) ChIP assays shown by Real-time PCR (D) and gel electrophoresis (E) showing the direct binding of MafB to the Cyclin D1 promoter region. The distal promoter region (-2000 bp -1800 bp) was used as a negative control. ${ }^{* *} P<0.01,{ }^{* * *} P<0.001$ between two groups. 
A

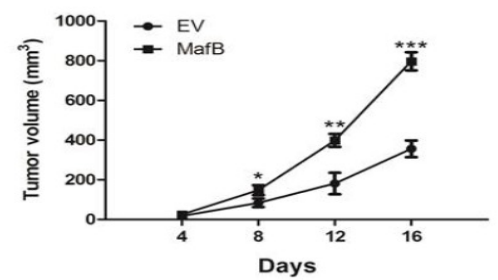

C

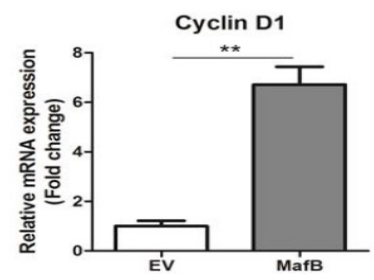

B

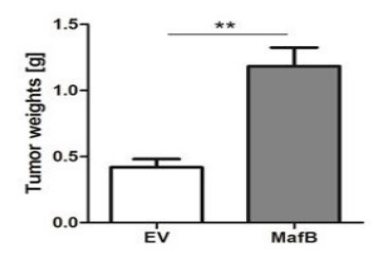

$\mathrm{D}$

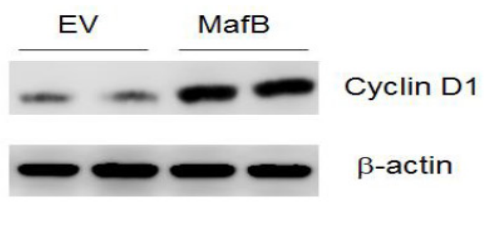

Fig. 6. MafB promotes HCC growth in vivo. (A-B) HepG2 cells stably transfected with adenovirus containing MafB or empty vector (NC) were injected into nude mice ( $\mathrm{n}=8$ for each group) and followed up for tumorigenesis. Growth curve of tumor volumes (A) and tumor weights (B) were taken 16 days after injection. (C-D) mRNA (C) and protein (D) levels of Cyclin D1 in tumors were determined by quantitative real-time PCR and western blots. ${ }^{*} P<0.05,{ }^{* *} P<0.01,{ }^{* * *} P<0.001$ between two groups.

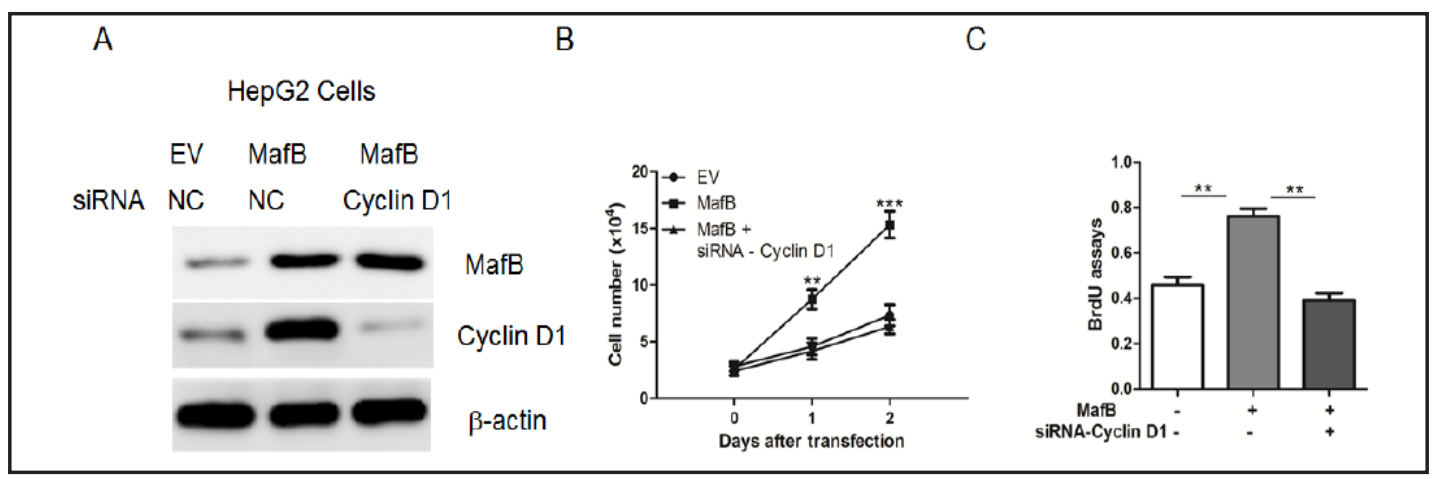

Fig. 7. Ablation of Cyclin D1 attenuates the proliferative effects of MafB. (A) Representative protein levels of Cyclin D1 and MafB in HepG2 cells. Cells were transfected with MafB overexpression plasmids or empty vector (EV) for $24 \mathrm{~h}$, and then transfected with siRNA oligos targeting Cyclin D1 or negative control (NC) for another 24 h. (B-C) Cell number (B) and proliferation (C) assays in HepG2 cells with MafB overexpression and (or) Cyclin D1 knockdown as indicated. ${ }^{* *} P<0.01,{ }^{* * *} P<0.001$ between two groups.

assays in HepG2 cells (Fig. 5D). Therefore, our results identify a functional MafB binding site in the Cyclin D1 promoter thereby mediating MafB-induced Cyclin D1 expression.

\section{Overexpression of MafB promotes tumor growth in vivo}

To determine the potential oncogenic effects of MafB in vivo, HepG2 cells transfected with adenovirus containing empty vector or MafB were injected subcutaneously to the skin under the front legs of the nude mice. Consistently, MafB overexpression significantly promoted cancer cell growth in mice, as shown by reduced tumor sizes and weight (Fig. 6A-6B). Furthermore, mRNA and protein levels of Cyclin D1 were also elevated in tumors overexpressing MafB (Fig. 6C-6D).

Knockdown of Cyclin D1 attenuated the proliferative roles of MafB

Finally, to confirm the relationship between MafB and Cyclin D1, HepG2 cells were administrated with siRNA oligos targeting Cyclin D1 or negative control, after transfection 


\section{Cellular Physiology Cell Physiol Biochem 2016;39:700-708 \\ \begin{tabular}{l|l|l} 
DOI: 10.1159/000445661 & $\begin{array}{l}\text { O 2016 The Author(s). Published by S. Karger AG, Basel } \\
\text { wwww.karger.com/cpb }\end{array}$
\end{tabular} \\ Yu et al.: MafB Promotes HCC Proliferation through Cyclin D1}

of MafB or empty vector (Fig. 7A). As shown in the Fig. 7B and 7C, knockdown of Cyclin D1 largely block the proliferative roles of MafB overexpression (Fig. 7B-7C), suggesting the oncogenic effects of MafB, at least in part, depend on its up-regulation of Cyclin D1.

\section{Discussion}

Better understanding the molecular mechanism of HCC could be helpful to identify novel prognostic markers or therapeutic targets for its treatment. In the present study, our data strongly support the notion that MafB acts as an oncogene in HCC progression. Firstly, MafB was up-regulated in both HCC tissues and cell lines. Secondly, ectopic overexpression of MafB was able to increase the HCC cell growth both in vitro and in vivo. On the other hand, MafB suppression inhibited HCC cell proliferation.

However, until now, the reason for the aberrant expression of MafB in HCC remains uncovered. Previous studies have shown that tumor necrosis factor (TNF) $\alpha$, a proinflammatory cytokine, increased MafB expression in human monocytic cells and primary adipocytes [7, 9, 13]. Besides, Borrelli et al. identified MafB as a direct transcriptional target of C/EBP $\delta$ in human primary keratinocytes by ChIP on chip assays [14]. Moreover, MafB expression was regulated by several MicroRNAs, including miR-148a and miR-223 [12, 1517]. Whether dys-regulation of these molecules contribute to the up-regulation of MafB in HCC remains to be determined in the future studies.

At the molecular level, our results found that MafB promoted HCC growth and progression through up-regulation of Cyclin D1. By luciferase reporter and ChIP assays, a MafB binding site was identified at the promoter region of human Cyclin D1 gene. More importantly, ablation of Cyclin D1 largely attenuated the proliferative roles of MafB in HCC cells, suggesting that the oncogenic function of MafB is, at least in part, dependent on its regulation of Cyclin D1. It has been well-established that Cyclin D1 accumulates to reach a maximum level before S-phase and regulates the G1/S-phase transition through binding and activating cyclin-dependent kinases (CDK4 and CDK6) [18, 19]. In addition, new roles of Cyclin D1 in tumorigenesis have been identified, such as the induction of chromosomal instability and cellular migration, enhancement of angiogenesis and inhibition of mitochondrial metabolism [20-23]. Therefore, whether MafB could regulate these biological events in cancer cells through Cyclin D1 will be determined in our future studies.

Taken together, these results indicated that MafB acts as an oncogene in HCC by induction of cell proliferation and growth. Given that a specific Cyclin D1-CDK4/6 inhibitor has obtained accelerated approval from the Food and Drug Administration for the treatment of certain types of human cancers [24, 25], our findings are encouraging and suggest that the MafB/Cyclin D1 regulatory pathway could be targeted for the future development of a treatment for HCC patients.

\section{Acknowledgements}

This work was partly supported by grants from the National Natural Science Foundation of China (81402473).

\section{Disclosure Statement}

None.

\section{References}

1 Li YW, Yang FC, Lu HQ, Zhang JS: Hepatocellular carcinoma and hepatitis B surface protein. World J Gastroenterol 2016;22:1943-1952. 


\section{Cellular Physiology Cell Physiol Biochem 2016;39:700-708 \begin{tabular}{l|l} 
and Biochemistry Published online: July 25, 2016 & $\begin{array}{l}\text { DO 2016 The Author(s). Published by S. Karger AG, Basel } \\
\text { www.karger.com/cpb }\end{array}$
\end{tabular} \\ Yu et al.: MafB Promotes HCC Proliferation through Cyclin D1}

2 Llovet JM, Villanueva A, Lachenmayer A, Finn RS: Advances in targeted therapies for hepatocellular carcinoma in the genomic era. Nat Rev Clin Oncol 2015;12:408-424.

3 Tsuchiya M, Misaka R, Nitta K, Tsuchiya K: Transcriptional factors, Mafs and their biological roles. World J Diabetes 2015;6:175-183.

4 Hang Y, Stein R: MafA and MafB activity in pancreatic beta cells. Trends Endocrinol Metab 2011;22:364373.

5 Kataoka K, Fujiwara KT, Noda M, Nishizawa M: MafB, a new Maf family transcription activator that can associate with Maf and Fos but not with Jun. Mol Cell Biol 1994;14:7581-7591.

6 Scoville DW, Cyphert HA, Liao L, Xu J, Reynolds A, Guo S, Stein R: MLL3 and MLL4 Methyltransferases Bind to the MAFA and MAFB Transcription Factors to Regulate Islet beta-Cell Function. Diabetes 2015;64:37723783.

7 Pettersson AM, Acosta JR, Bjork C, Kratzel J, Stenson B, Blomqvist L, Viguerie N, Langin D, Arner P, Laurencikiene J: MAFB as a novel regulator of human adipose tissue inflammation. Diabetologia 2015;58:2115-2123.

8 Abdellatif AM, Ogata K, Kudo T, Xiafukaiti G, Chang YH, Katoh MC, El-Morsy SE, Oishi H, Takahashi S: Role of large MAF transcription factors in the mouse endocrine pancreas. Exp Anim 2015;64:305-312.

9 Gemelli C, Zanocco Marani T, Bicciato S, Mazza EM, Boraschi D, Salsi V, Zappavigna V, Parenti S, Selmi T, Tagliafico E, Ferrari S, Grande A: MafB is a downstream target of the IL-10/STAT3 signaling pathway, involved in the regulation of macrophage de-activation. Biochim Biophys Acta 2014;1843:955-964.

10 Kim K, Kim JH, Lee J, Jin HM, Kook H, Kim KK, Lee SY, Kim N: MafB negatively regulates RANKL-mediated osteoclast differentiation. Blood 2007;109:3253-3259.

11 Vicente-Duenas C, Romero-Camarero I, Gonzalez-Herrero I, Alonso-Escudero E, Abollo-Jimenez F, Jiang X, Gutierrez NC, Orfao A, Marin N, Villar LM, Criado MC, Pintado B, Flores T, Alonso-Lopez D, De Las Rivas J, Jimenez R, Criado FJ, Cenador MB, Lossos IS, Cobaleda C, Sanchez-Garcia I: A novel molecular mechanism involved in multiple myeloma development revealed by targeting MafB to haematopoietic progenitors. EMBO J 2012;31:3704-3717.

12 Yang W, Lan X, Li D, Li T, Lu S: MiR-223 targeting MAFB suppresses proliferation and migration of nasopharyngeal carcinoma cells. BMC Cancer 2015;15:461.

13 Zhang Y, Chen Q Ross AC: Retinoic acid and tumor necrosis factor-alpha induced monocytic cell gene expression is regulated in part by induction of transcription factor MafB. Exp Cell Res 2012;318:24072416.

14 Borrelli S, Fanoni D, Dolfini D, Alotto D, Ravo M, Grober OM, Weisz A, Castagnoli C, Berti E, Vigano MA, Mantovani R: C/EBPdelta gene targets in human keratinocytes. PLoS One 2010;5:e13789.

15 Cheng P, Chen C, He HB, Hu R, Zhou HD, Xie H, Zhu W, Dai RC, Wu XP, Liao EY, Luo XH: miR-148a regulates osteoclastogenesis by targeting $\mathrm{V}$-maf musculoaponeurotic fibrosarcoma oncogene homolog $\mathrm{B}$. J Bone Miner Res 2013;28:1180-1190.

16 Gu S, Cheung HH, Lee TL, Lu G, Poon WS, Chan WY: Molecular mechanisms of regulation and action of microRNA-199a in testicular germ cell tumor and glioblastomas. PLoS One 2013;8:e83980.

17 Tang P, Xiong Q, Ge W, Zhang L: The role of microRNAs in osteoclasts and osteoporosis. RNA Biol 2014;11:1355-1363.

18 Baldin V, Lukas J, Marcote MJ, Pagano M, Draetta G: Cyclin D1 is a nuclear protein required for cell cycle progression in G1. Genes Dev 1993;7:812-821.

19 Matsuda Y: Molecular mechanism underlying the functional loss of cyclindependent kinase inhibitors p16 and p27 in hepatocellular carcinoma. World J Gastroenterol 2008;14:1734-1740.

20 Pestell RG: New roles of cyclin D1. Am J Pathol 2013;183:3-9.

21 Li Z, Jiao X, Wang C, Ju X, Lu Y, Yuan L, Lisanti MP, Katiyar S, Pestell RG: Cyclin D1 induction of cellular migration requires p27(KIP1). Cancer Res 2006;66:9986-9994.

22 Wang C, Li Z, Lu Y, Du R, Katiyar S, Yang J, Fu M, Leader JE, Quong A, Novikoff PM, Pestell RG: Cyclin D1 repression of nuclear respiratory factor 1 integrates nuclear DNA synthesis and mitochondrial function. Proc Natl Acad Sci U S A 2006;103:11567-11572.

23 Petty WJ, Dragnev KH, Dmitrovsky E: Cyclin D1 as a target for chemoprevention. Lung Cancer 2003;41:S155-161.

24 Schroder LB, McDonald KL: CDK4/6 Inhibitor PD0332991 in Glioblastoma Treatment: Does It Have a Future? Front Oncol 2015;5:259.

25 Li T, Zhao X, Mo Z, Huang W, Yan H, Ling Z, Ye Y: Formononetin promotes cell cycle arrest via downregulation of Akt/Cyclin D1/CDK4 in human prostate cancer cells. Cell Physiol Biochem 2014;34:1351-1358. 